\title{
Defining viable recovery paths toward sustainable fisheries
}

\author{
Vincent Martinet $^{\mathrm{a},{ }^{*}}$, Olivier Thébaud ${ }^{\mathrm{a}, \mathrm{b}}$, Luc Doyen $^{\mathrm{c}}$ \\ ${ }^{a}$ Marine Economics Department, IFREMER, ZI Pointe du Diable BP 70, 29280 Plouzané, France \\ bUBO-CEDEM, France \\ ${ }^{\mathrm{c} C N R S}$, CERSP, Muséum National d'Histoire Naturelle, France \\ *: Corresponding author : V. Martinet, email address : vincent.martinet@grignon.inra.fr
}

\begin{abstract}
:
This paper develops a formal analysis of the recovery process for a fishery, from crisis situations to desired levels of sustainable exploitation, using the theoretical framework of viable control. We define sustainability as a combination of biological, economic and social constraints which need to be met for a viable fishery to exist. Biological constraints are based on the definition of a minimum resource stock to be preserved. Economic constraints relate to the existence of a guaranteed profit per vessel. Social constraints refer to the maintenance of a minimum size of the fleet, and to the maximum speed at which fleet adjustment can take place. Using fleet size adjustment and fishing effort per vessel as control variables, we first identify the states of this bioeconomic system for which sustainable exploitation is possible, i.e. for which all constraints can be dynamically met. Such favorable states are called viable states. We then examine possible transition phases, from non-viable to viable states. We characterize recovery paths with respect to the time of crisis of the trajectory, which is the number of periods during which the constraints are not respected. The approach is applied to the single stock of the bay of Biscay Nephrops fishery. The transition path identified through the viability approach is compared to the historical recovery process, and to both open-access and optimal harvesting scenarios.
\end{abstract}

Keywords: Sustainable fishing; Recovery; Fishery policies; Bio-economic modeling 


\section{Introduction}

According to recent studies, the maximum production potential of marine fisheries worldwide was reached at least two decades ago; since then, due to the widespread development of excess harvesting capacity, there has been an increase in the proportion of marine fish stocks which are exploited beyond levels at which they can produce their maximum (FAO, 2004; Garcia and Grainger, 2005). Hence, the problem of managing fisheries is increasingly cast in terms of restoring them to higher sustainable levels of fish stocks, catches, and revenues from fishing.

The problems posed by fisheries restoration are dynamic in nature: beyond the issue of choosing adequate objective levels for restored fisheries, a key question is the identification and the selection of the possible paths towards these objective levels. In practical situations, this question is crucial as it relates to the feasibility (technical, economic, biological) and to the social and political acceptability of the adjustments required for fisheries to be restored, hence to the actual feasibility of driving fisheries back towards decided sustainability objectives.

The definition of preferred strategies for the harvesting of marine fish stocks has been widely studied in the literature on renewable resource management. While most of the initial work focused on the comparative statics of the problem, analysis of the dynamics of bio-economic systems has developed as a substantial body of literature. In the domain of fisheries, early studies focused on the dynamics of open access and competitive harvesting of common pool resources (Smith, 1968; Wilen, 1976), and on the means to optimally drive such dynamic bioeconomic systems towards a given stationary state (Clark and Munro, 1975; Clark, 1976, 1985), taking into account issues of capital malleability (Clark et al., 1979; McKelvey, 1985), and uncertainty (Beddington and May, 1977; Hilborn and Walters, 1992). Developments have been largely theoretical, and are based on a single command variable such as fishing effort, and a single optimization criterion such as the net present value of the expected benefits derived from harvesting. In some cases, data concerning the dynamics of specific fisheries has been used to estimate the parameters of discrete-time simulation models of the dynamics of these fisheries (Bjorndal and Conrad, 1987; Ward and Sutinen, 1994; Homans and Wilen, 1997). Such models have been used for the simulation of specific adjustment trajectories for given bioeconomic systems, according to predetermined scenarii, and on their a posteriori evaluation with respect to a given set of criteria, e.g. economic, social and/or biological (Smith, 1969; Holland, 2000; Mardle and Pascoe, 2002).

In this article, we develop a formal analysis of the recovery paths for a fish- 
ery, based on viable control theory. The viable control framework (Aubin, 1991) focuses on intertemporal feasible paths. This consists in the definition of a set of constraints that represents the "good health" of the system at any moment, and in the study of conditions which allow these constraints to be satisfied along time. More specifically, in the context of renewable resource harvesting, viability may imply the satisfaction of both economic and ecological constraints. In this sense, it is a multi-criteria approach sometimes referred to as a "co-viability" approach. Moreover, an intergenerational equity feature is naturally integrated within this framework (Martinet and Doyen, 2007). From the ecological viewpoint, the so-called population viability analysis (PVA) (Morris and Doak, 2003) and conservation biology have concerns close to viable control by focusing on extinction processes generally within a stochastic framework. Cury et al. (2005) advocate the use of the viability framework as part of the ecosystem approach to fisheries, and it has been proposed as a useful basis for the analysis of renewable resources management (Béné and Doyen, 2000; Béné et al., 2001; Doyen and Béné, 2003; Eisenack et al., 2006; De Lara et al., in press).

In particular, the viable control approach allows characterization of the dynamics of a fishery in terms of its capacity to remain within pre-defined constraints, beyond which its continued long-term existence would be jeopardized. We adopt this characterization, and define constraints on a fishery related to micro-economic, biological and social factors. Any path that does not respect the ecological, economic or social constraint (or any combination of these) will be associated to a crisis of the fishery. Following Béné et al. (2001), we first use the mathematical concept of viability kernel to identify the set of states of the fishery from which it is possible to satisfy these constraints dynamically and thus to avoid crisis. This kernel represents the "target" states for a perennial fishery. In a second step, our analysis focuses on crisis situations, outside the viability kernel. In particular, the attention is paid to the ways by which the fishery can recover from such crisis, and on the 'recovery path' leading the system back to sustainable states lying in the viability kernel. We use the concept of time of crisis introduced in Doyen and Saint-Pierre (1997) to define the horizon over which such targets can be reached, and examine transition paths with respect to that time of crisis. More specifically, the minimum time of crisis with respect to possible decision paths is studied, revealing the shortest path(s) to a viable state. Such an optimal control problem provides joint information on viability, restoration and irreversibility issues. Consequently it sheds an interesting light on the sustainability issues of the bio-economic model. However, this minimal time of crisis is not a usual optimal control problem. It turns out to be difficult to solve mathematically and here numerical approximations are required as in Béné et al. (2001).

The analysis is applied to a simplified representation of the Bay of Biscay (ICES area VIII) nephrops fishery, and focuses on the historical changes as 
observed via this simple model, estimated using available data. We discuss the viability and recovery of various trajectories, including the estimated historical trajectory, and simulated alternative trajectories. Numerical analysis has been computed in Scilab code. ${ }^{1}$

The article is structured as follows. The fishery is described in section 2 . The simplified model of the bay of Biscay nephrops fishery used for the analysis is presented (2.1). The economic, biological and social constraints determining the viability of the fishery are defined (2.2), and the case study is described (2.3). In section 3 , we develop the theoretical framework that allows us to analyse the conditions under which the viability constraints can be satisfied throughout time (3.1), and to study recovery processes from crisis situations (3.2). We use this framework in section 4 to study possible recovery paths from a historical crisis situation with respect to the estimated historical trajectory. Section 5 concludes.

\section{Defining a sustainable fishery}

\subsection{A bio-economic model of the fishery}

In this paper, we consider a single stock fishery, characterized for each year $t$ by a biomass $B_{t}$ of the exploited resource stock and a size of the fleet $K_{t}$. The dynamics of the bio-economic system are controlled by the effort $e_{t}$ corresponding to the days at sea per period and per vessel and the change in fleet size $\xi_{t}$, namely the number of boats entering or exiting the fleet.

We use a discrete time version of the "logistic model" (Schaefer, 1954) to represent the fish stock's renewal function. The growth of the resource stock is given by

$$
R\left(B_{t}\right)=r B_{t}\left(1-\frac{B_{t}}{B_{\text {sup }}}\right),
$$

where $B_{\text {sup }}$ is the carrying capacity of the ecosystem for the resource stock.

The fleet is assumed homogeneous. Each vessel has the same access to the resource and the same technical characteristics. Global catches are defined by

$$
C_{t}=q B_{t} e_{t} K_{t},
$$

where $q$ represents the catchability of the resource. The dynamics of the resource, combining eq. (1) and (2), can thus be described, following Gordon

$\overline{1}$ Information about this software of scientific calculus and free download are available at http://www.scilab.org/. 
(1954), by

$$
B_{t+1}=B_{t}+R\left(B_{t}\right)-C_{t}=B_{t}+r B_{t}\left(1-\frac{B_{t}}{B_{\text {sup }}}\right)-q B_{t} e_{t} K_{t}
$$

The economic status of the fleet is characterized by per vessel profit. This profit depends on landings $L_{t}$ of the resource, defined with respect to the per vessel catches $c_{t}=C_{t} / K_{t}=q B_{t} e_{t}$ and a discard rate $\tau_{d}$

$$
L_{t}=\left(1-\tau_{d}\right) q B_{t} e_{t}
$$

Based on these landings, gross return associated to the targeted species is defined as a part $\lambda$ of the vessel's total gross return. ${ }^{2}$ Vessel profit thus reads

$$
\pi_{t}=\left(p\left(1-\tau_{d}\right) q B_{t} e_{t}\right) \frac{1}{\lambda}-\left(\omega_{f}+\omega_{v} e_{t}\right)
$$

where $p$ is an exogenous resource price that is considered constant. $\omega_{f}$ represents fixed costs and $\omega_{v}$ a per effort unit cost. Thus defined, fleet profit represents the remuneration of production factors (capital and labor) at vessel level.

The production structure is assumed to be slowly flexible, in terms of both capital and labor. The size of the fleet evolves according to a decision control $\xi_{t}$,

$$
K_{t+1}=K_{t}+\xi_{t} .
$$

A degree of capital inertia is assumed to exist in the fishery. Due to technical and regulatory constraints, a maximum number $\xi_{\text {sup }}$ of vessels can enter the fishery in any time period. Also, the number of vessels exiting the fleet in any time period can not exceed $\xi_{\text {inf }}$, due mainly to social and political constraints (see below). Such rigidities are captured by condition

$$
-\xi_{\text {inf }} \leq \xi_{t} \leq \xi_{\text {sup }}
$$

On the other hand, fleet activity (effort per period $e_{t}$ ) can change, and even be set to nil. Moreover, the effort is bounded by a maximum number of days at sea per period ${ }^{3} e_{\text {sup }}$. Hence the technical constraint on vessel effort:

$$
0 \leq e_{t} \leq e_{\text {sup }}
$$

$\overline{2}$ Taking $\lambda=1$ means that the species studied is the only one exploited by the fleet.

3 Which obviously cannot exceed 365 days per year (366 for leap years), but will usually be lower. 


\subsection{Defining viability constraints}

We define the viability of the fishery by a set of biological, economic and social constraints that have to be respected throughout time.

Biological constraint: In order to preserve the renewable resource, a minimum resource stock $B_{\min }$ is considered.

$$
B_{t} \geq B_{\min }
$$

Economic constraint: An economic constraint on vessel performance is also considered: profit per vessel is required to be greater than a threshold $\pi_{\min }$ for economic units to be viable.

$$
\pi_{t} \geq \pi_{\min }
$$

This minimum profit is defined such as to ensure remuneration of both capital and labor at their opportunity costs.

This minimum profit constraint actually leads to induced constraints on a minimal effort $\underline{e}(B)$ and a minimal stock size $\underline{B}$, for any given fleet size, as proven in the appendix A.2, p.19.

Social constraint: To take into account social concerns, the viability of the fishery is described by a constraint on fleet size. We require the number of vessels to be greater than a threshold $K_{\min }$ :

$$
K_{t} \geq K_{\min }
$$

ensuring a minimum employment and activity in the fishery.

In addition to this minimum fleet size, we assume that the speed at which fleet size can be reduced is also limited. The constraint on the adjustment possibilities regarding the fleet size (eq. 7) can be interpreted as a social and political constraint limiting the number of vessels (and employment) leaving the fleet during any time period. This interpretation is somewhat different from that encountered in the literature regarding capital inertia, which is assumed to result mainly from the lack of possibilities to quickly reallocate specific fishing assets to alternative uses, a technical, rather than social constraint. 


\subsection{The case of a simplified model for the Bay of Biscay Nephrops fishery}

To illustrate the method and type of results produced, the analysis is applied to a simplified model of the Bay of Biscay Nephrops fishery (ICES area VIII). The empirical modeling approach, based on the estimation of a surplus production model, was chosen with the aim to limit analytical and computational difficulties. This provides a reasonable representation of the qualitative dynamics of the fishery over the past ten years, which is then used to apply the viability framework of analysis. However, it ignores certain important characteristics of the fishery, in particular the age structure of the population and the uncertainty in recruitment, which limits the usefulness of the model for policy recommendations. Including such factors in the analysis requires the development of a more realistic model of the biological component of this fishery.

Appendix A.1 describes how parameters have been estimated and provides the parameter values and constraint levels used in the analysis. In particular, based on 2003 data, it appears that the minimum profit per vessel $\pi_{\min }$ in our stylized model of the fishery is 130,000 euros per year. ${ }^{4}$ The minimum stocks size $B_{\min }$ was fixed at 5,000 tons based on the available biological reference point defined in the literature for the resource stock. The minimal fleet size $K_{\min }$ was arbitrarily fixed at 100 vessels, and the maximum speed of entry/exit of vessels in the fleet (respectively $\xi_{\text {sup }}$ and $\xi_{\text {inf }}$ ) at 10 per year.

In 2003, the fleet was composed by 235 vessels with an average profit of 165,000 euros. The resource stock was estimated at about 18,600 tons. The catches were estimated at 5,769 tons. Viability constraints, as defined in the appendix, were thus met for that particular year. However, in the early 90's, the resource stock decreased, and the associated per-vessel profit as estimated via the model dropped below the economic viability constraint. The estimated resource stock reached its lowest level in 1994, at about 14,000 tons, with an estimated pervessel profit at 78,000 euros. The fishery thus faced a crisis period (at least from an economic point of view), from which it appears to have recovered since then.

4 This level corresponds to the actual (mean) profit required to ensure the remuneration of production factors (labor and capital) at their opportunity costs in the Bay of Biscay Nephrops fishery, as measured via the economic surveys carried out by Ifremer. 


\section{Characterizing viable situations and crisis situations}

\subsection{The viability kernel and viable harvesting strategies}

The aim of this section is to define state configurations for the fishery, including resource stock and fleet size, which are compatible with the viability constraints which have been defined. The question is to determine whether the dynamics are compatible with the set of constraints. For this purpose, we use the viable control approach and study the consistence between dynamics (3) and (6) and the constraints (7), (9), (10) and (11). The set of bioeconomic states from which there exist intertemporal paths respecting the entire set of constraints is called the viability kernel of the problem. We associate it with the sustainable exploitation configurations.

Viable states: Formally, for our problem, the viability kernel is defined by

$$
\text { Viab }=\left\{\begin{array}{l|l}
\left(B_{0}, K_{0}\right) & \begin{array}{l}
\exists(e(.), \xi(.)) \text { and }(B(.), K(.)), \text { starting from }\left(B_{0}, K_{0}\right) \\
\text { satisfying dynamics }(3) \text { and }(6) \\
\text { and constraints }(7),(9),(10) \text { and }(11) \text { for any } t \in \mathbb{N}^{+}
\end{array}
\end{array}\right\} .
$$

The viability kernel Viab for our simplified model of the nephrops fishery (using parameter values presented in appendix A.1) is represented in Fig. 1. The estimated historical trajectory of the fishery is also displayed. Note that the situation in 1994, as defined via the model, is not viable in the sense that it does not belong to the viability kernel.

Fig. 1 also presents the estimated position of specific 'reference points' for the fishery, including the states compatible with a Maximum Sustainable Yield (MSY), the Open Access Equilibrium (OAE), and the Maximum Economic Yield (MEY). These states belong to the broader set of viable stationary states of the bioeconomic system, which is also represented in the figure: this set is composed of all the states between the decreasing straight line and the curved line (see appendix A.3 for a formal description of stationary states).

For any given initial state $\left(B_{0}, K_{0}\right)$ in the viability kernel, there exists at least one intertemporal set of decisions $(e(),. \xi()$.$) for which the associated$ trajectory starting from $\left(B_{0}, K_{0}\right)$ respects all the constraints forever. Note that several viable decisions may exist. Another important point is that all a priori admissible decisions are not necessarily viable and that some of them may lead the system outside the viability kernel, i.e. induce exploitation patterns that do not respect all the viability constraints simultaneously. For example, the 


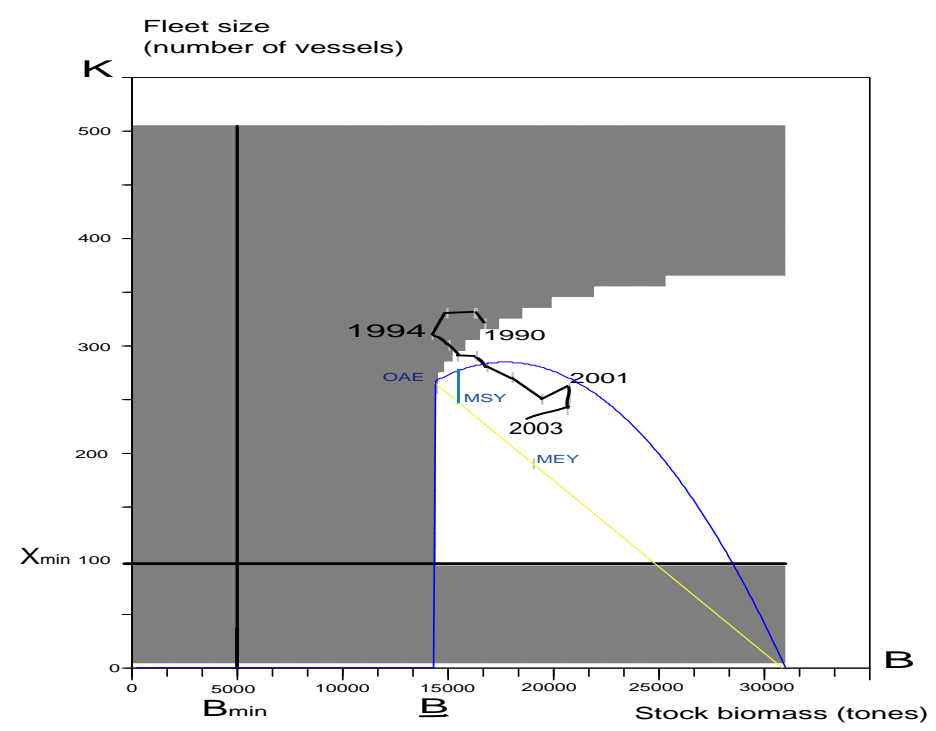

Fig. 1. The viability kernel, stationary states and the historical dynamics as estimated via the model.

estimated historical dynamics of the fishery show that it entered the viability kernel in 1996. This means, from that year, it would have been possible to follow a viable exploitation trajectory, in the sense that there were admissible decisions such that all of the constraints could have been met in the following years. Nevertheless, harvesting decisions from 1996 onwards were not viable, since the profit constraint was not met, according to the model. ${ }^{5}$

\subsection{Outside the kernel: crisis situations}

The viability kernel represents the "goal" for recovery paths starting from initial states outside the kernel, i.e. the set of states the system must reach to make a viable exploitation path possible. From its very definition, from any initial state outside the kernel, there are no decisions that make it possible to satisfy the constraints in the long run. At least one of the constraints will be violated in a finite time, whatever the decisions are. The system thus faces a crisis situation if either the bioeconomic state is located outside the kernel, or the intertemporal path is bound to leave the kernel in the following years.

To recover from such crisis situations, fleet size and effort per vessel must be adjusted so that the bioeconomic system re-enters the viability kernel. This will only be possible if the chosen decisions lead to relax at least one of the constraints during a certain period of time, in order for the system to recover.

5 See appendix A.1 for the data describing the estimated historical path followed by the fishery. 
Minimum time of crisis: Our study addresses the ways by which the fishery can recover from such crisis, and on the 'recovery paths' leading the system back to sustainable states. Following Doyen and Saint-Pierre (1997) or Béné and Doyen (2000), we define the time of crisis as the time spent outside the viability constraints by a trajectory. A transition phase can then be characterized by a time of transition, corresponding to this length of time. Starting from a given bioeconomic state, various transition phases can exist, that reach the viability kernel more or less quickly. Of particular interest is the minimal time of crisis which exhibits the decision strategy that achieves the shortest time of crisis. More formally, the optimal control problem associated with the minimum time of crisis, i.e. the minimum time spent outside the viability constraints by trajectories starting from $\left(B_{0}, K_{0}\right)$, is defined by

$$
\mathcal{C}\left(B_{0}, K_{0}\right)=\inf _{\substack{(B(.), K(.), e(.), \xi(.)) \\ \text { admissible path }}} \sum_{t=0}^{\infty} \mathbb{I}\left(B_{t}, K_{t}, e_{t}, \xi_{t}\right)
$$

where

- II, the characteristic function that counts the number of period for which viability constraints do not hold true, is defined by

$$
\mathbb{I}(B, K, e, \xi)= \begin{cases}0 & \text { if }(B, K, e, \xi) \text { satisfy constraints }(9),(10) \text { and (11) } \\ 1 & \text { otherwise }\end{cases}
$$

- path $(B(),. K(),. e(),. \xi()$.$) is said to be admissible whenever it satisfies dy-$ namics and control constraints $(3),(6)$ and $(7)$ while starting from $\left(B_{0}, K_{0}\right)$.

As explained in Doyen and Saint-Pierre (1997), this minimum time of crisis approach allows to simultaneously deal with viability and target ${ }^{6}$ problems. In this sense, this optimal control problem provides information on the potential sustainability of the system. Hence, the set of the states for which the minimum time of crisis is lower than or equal to time $T$ is said to be viable at scale $T$. In particular, the viability kernel defined in the previous section corresponds to a 0 minimal time of crisis (viability at scale 0 ). In an opposite way, a very informative case related to irreversibility issues occurs whenever the minimal time of crisis is infinite (or very large). Moreover, it turns out that the minimal time of crisis can be related to the time spent outside the viability kernel and to the shortest transition path towards the viability kernel. Regarding this, the minimum time of crisis expands the viability approach and inherits most of its advantages which includes the multi-criteria (bio-economic

\footnotetext{
$\overline{6}$ In fact, problems known as "minimal time" or "minimal time to hit a target". It is mainly used in robotics and engineering science.
} 
here) perspective. However, it is worth pointing out that such an optimal control problem is not usual and turns out to be difficult to solve mathematically. One major technical reason for that relies on the fact that it is a "degenerated" (non regular) optimal control problem in the mathematical sense as the intertemporal criterion depends on a characteristic function $\mathbb{I}$ which displays non smooth features (0 or 1 Boolean values). Thus, numerical methods, based for instance on dynamic programming, are required to approximate this value function and the associated optimal controls.

Such a numerical approach is applied here to the model of nephrops fishery. The level sets of this value function $\mathcal{C}(.,$.$) are represented in Fig. 2$.

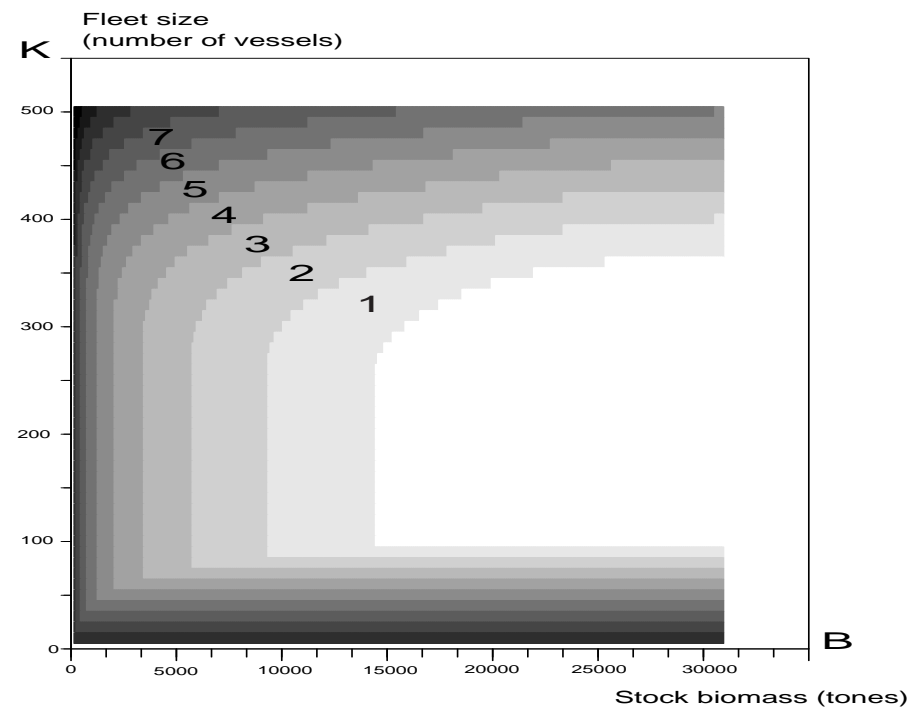

Fig. 2. Minimum time of crisis $\mathcal{C}\left(B_{0}, K_{0}\right)$ and scale of viability.

\section{Recovering from a crisis situation}

In this section, we characterize recovery processes from crisis situations to viable situations. As an example for the analysis, we use the estimated status of the fishery in 1994 as the initial state from which recovery paths can be discussed. As mentioned previously, the situation of the fishery as estimated via the model for that year appears critical as it corresponds to the farthest estimated state from the viability kernel (lowest estimated stock and per vessel profit). From this crisis situation, the fishery has recovered according to an estimated path, which can be compared to various paths defined a priori. We distinguish three paths:

- open access fishery; 
- (economically) optimal intertemporal harvesting;

- minimum time of crisis.

\subsection{The three scenarii}

\subsubsection{Open access}

The open access case corresponds to a situation in which vessels can freely enter and exit the fishery, subject to the inertia constraints (7) described above, and choose their individual effort level. ${ }^{7}$ In that case, as claimed in lemma 3 (appendix A.2, p.20), the individual effort will be maximum: the individual optimal behavior is to have the maximum admissible effort, which reads

$$
e_{t}^{O A}=e_{\text {sup }}
$$

We consider that, if individual profit is greater than the minimum profit $\pi_{\min }$, the fleet size increases as new vessels enter the fishery, leading to rent dissipation. On the contrary, if the individual profit is lower than the $\pi_{\min }$ level, vessels leave the fleet as fishing activity does not cover opportunity costs of production factors. In our representation of the Open Access regime, the dynamics of capital, i.e. that of the fleet size, can be described as follows

$$
\xi_{t}^{O A}=\left\{\begin{array}{lll}
\xi_{\mathrm{sup}} & \text { if } & \pi_{t} \geq \pi_{\mathrm{min}} \\
-\xi_{\mathrm{inf}} & \text { if } & \pi_{t}<\pi_{\mathrm{min}}
\end{array}\right.
$$

\subsubsection{Discounted dynamic maximum economic yield}

As a second scenario, we consider a regulated fishery where the decision maker optimizes the discounted intertemporal profit of the fleet.

At fleet level, the optimal behavior is determined by maximizing the intertemporal sum of discounted fleet profits, with respect to the allocation of the fishing effort through time and the management of the fleet size, which reads

$$
\max _{e(.), \xi(.)} \sum_{t=0}^{\infty} \frac{1}{(1+\delta)^{t}} K_{t}\left(p q B_{t} e_{t}-\left(\omega_{f}+\omega_{v} e_{t}\right)\right)
$$

where $\delta$ represents the social discount rate or, from a microeconomic perspective, the opportunity cost of capital. ${ }^{8}$ In the general framework, the optimal

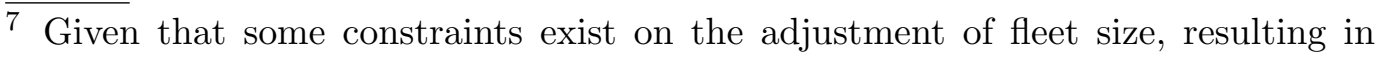
particular from regulations and social norms, this path could more precisely be described as a case of regulated open access.

8 For the numerical application, the interest rate is set to $5 \%$. 
solution of such a problem (Clark, 1990) is to reach an optimal steady state following a "bang-bang" strategy (or most rapid approach). In the case presented here, there is no "bang-bang" strategy, given the inertia in capital (fleet size) adjustment.

\subsubsection{Minimum time of crisis}

The two harvesting scenarios considered above lead to paths that do not take the viability constraints into account. If these constraints apply, it is possible that some of the trajectories represented above may actually lead to situations of crisis due to a collapse of the stock, the economic extinction of the fishery, or to social unrest associated with the adjustment paths considered. Each time that a viability constraint is not respected, the fishery is about to collapse, unless some external help occurs (financial support when the economic constraint is not achieved, or social compromise when the fleet size adjustment is too fast $\left.^{9}\right)$.

Adjustment towards viable states of the fishery should also be characterized in terms of the capacity of intertemporal recovery paths to respect the constraints defined in section 2.2. An adjustment path of particular interest is thus the one that ensures a minimum time of crisis as defined in the previous section. We thus compute the minimum time of crisis associated with the 1994 bioeconomic state, and the recovery path that minimizes the time of crisis. ${ }^{10}$

\subsection{Compared trajectories}

The computed trajectories are presented in Fig. 3 and 4. Fig. 3 (a) shows the evolutions of the resource stock $B_{t}$ while the fleet size $K_{t}$ is depicted in Fig. 3 (b). The paths of fishing effort $e_{t}$ corresponds to Fig. 4(a) and the per vessel profit $\pi_{t}$ is displayed on Fig. 4(b).

Based on the model developed, the historical dynamics of the Nephrops fishery from the 1994 situation (lowest estimated biomass) are characterized by a strong reduction of the fleet size, along with a recovery of the resource stock. From 1994 to 2001, profit was lower than the viability threshold $\pi_{\min }=$ 130, 000 euros. During these years, even if the bioeconomic state reached the viability kernel quickly (and thus would have made it possible to satisfy the profit constraint), profit remained low. The time of crisis, as it has been de-

9 Usually, vessels are retired from the fishery with financial compensation.

${ }^{10}$ In this simulation, we strengthen constraint (7) and limit the speed of the fleet size adjustment to 5 boats per year, which appears to be a "softer" adjustment speed than that observed for the historical path. 


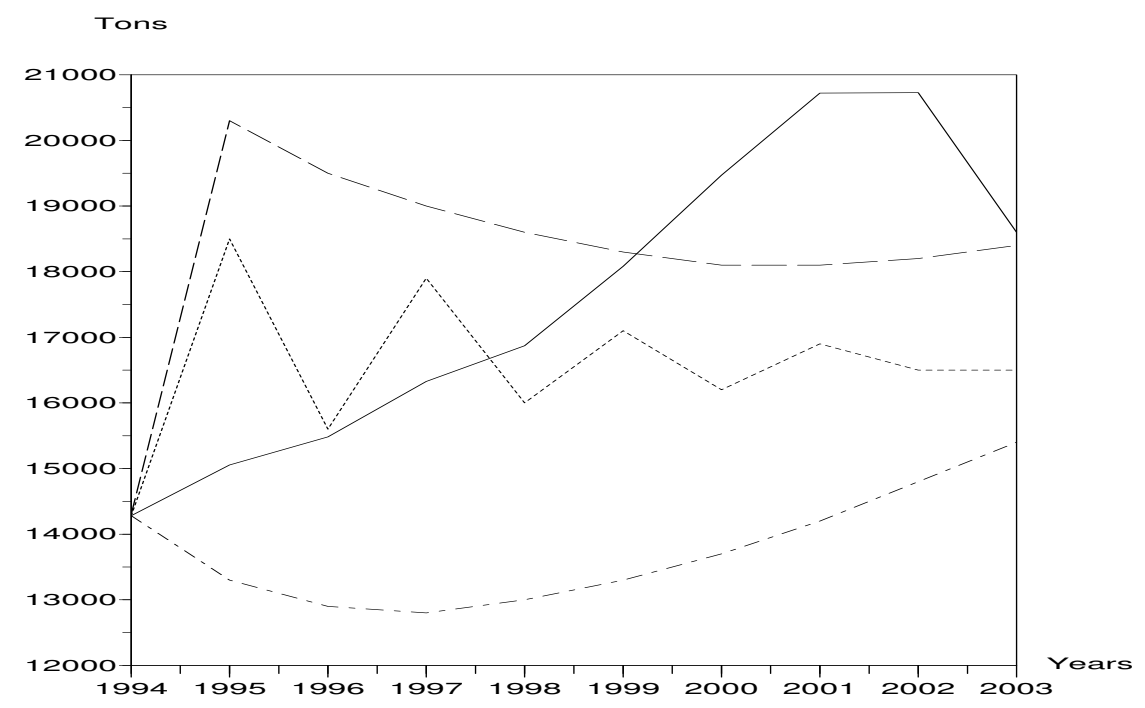

(a) Resource stock biomass $B_{t}$

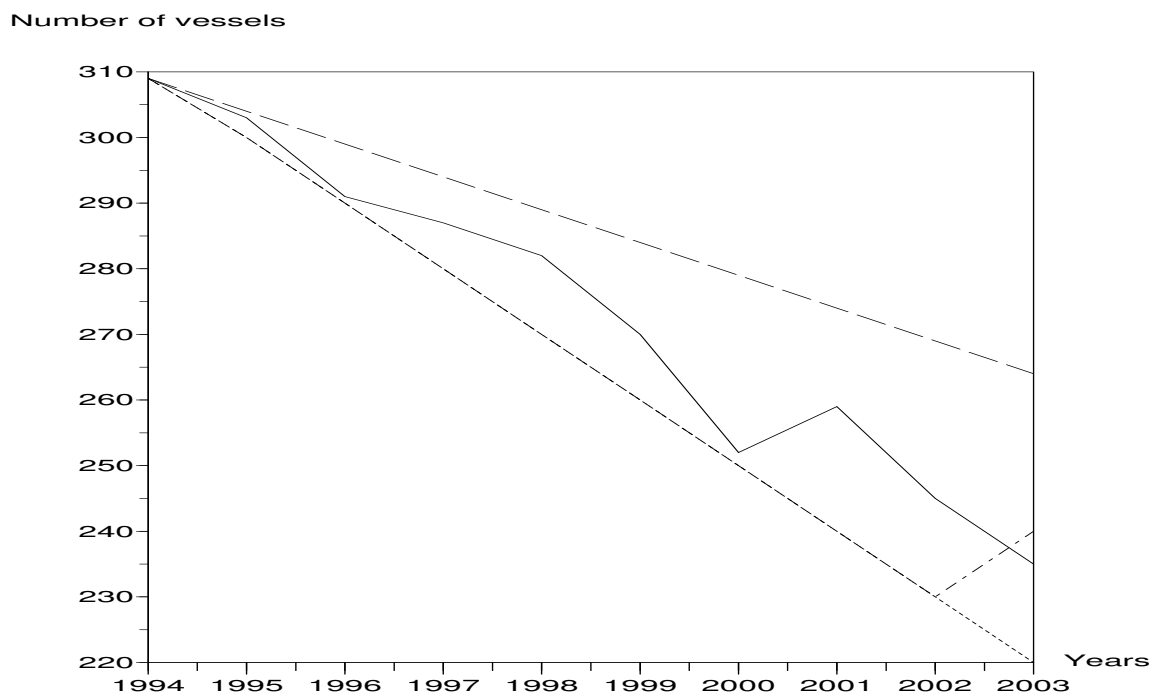

(b) Fleet size $K_{t}$

Fig. 3. Recovery trajectories (and the historical path) from the estimated 1994 crisis situation.

Historical path

- - - - Open Access regime

- - - - - - Optimal Economic Intertemporal path

_ _ _ Minimal Time of Crisis

fined in section 3.2, associated to the historical path starting from the 1994 bioeconomic state, is then 7 .

It appears that an open access exploitation from the 1994 situation would have 


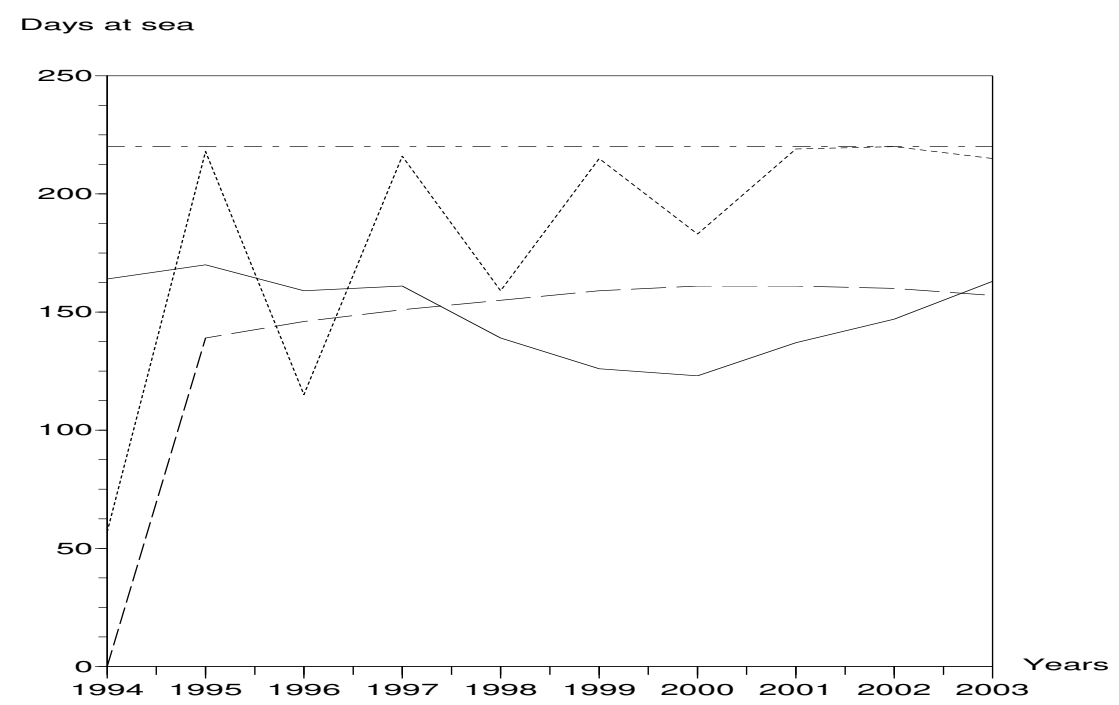

(a) Per vessel effort $e_{t}$

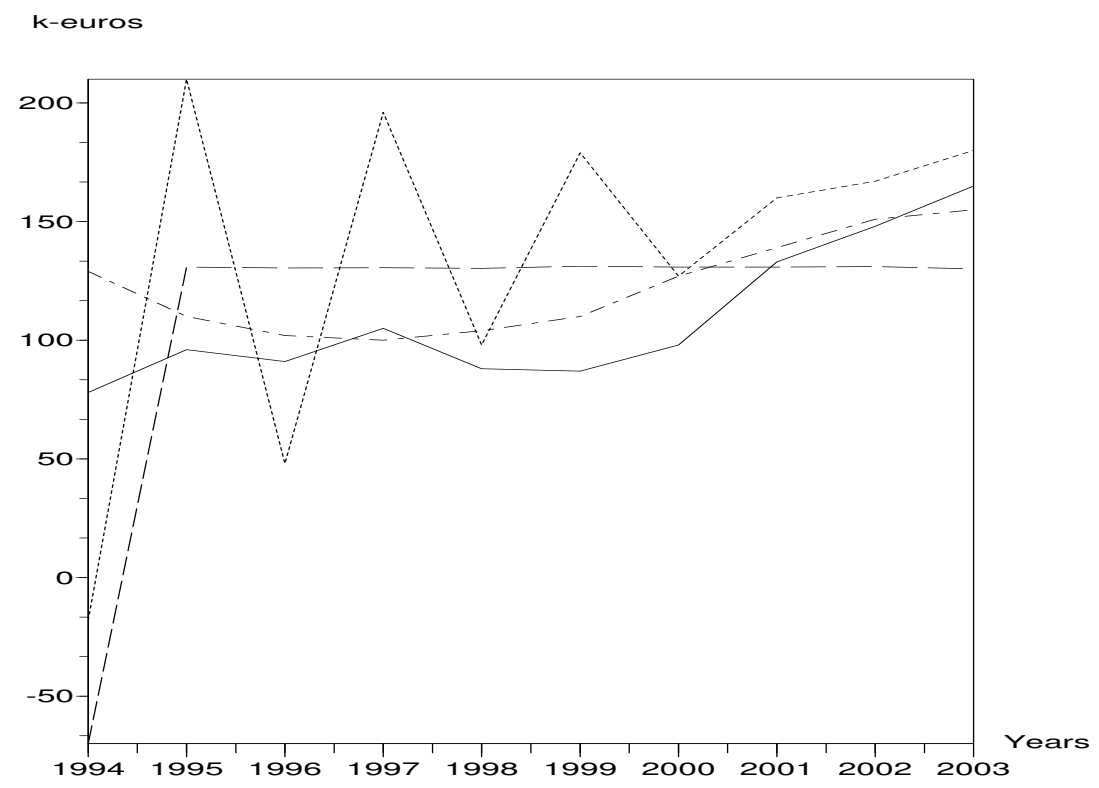

(b) Per vessel profit $\pi_{t}$

Fig. 4. Recovery trajectories (and the historical path) from the 1994 crisis situation. Historical path

- - - - Open Access regime

- - - - - - Optimal Economic Intertemporal path

_ — - Minimal Time of Crisis

led to an initial decrease of the resource stock, the fleet size and the per vessel profit, with a recovery at the end of the simulation period. The associated time of crisis is 7 years, which is the same as the historical trajectory. 
Maximizing the intertemporal economic profit would have led to an alternance of high and low exploitation levels, tending towards the stationary state characterizing the Maximum Economic Yield. The associated time of crisis is 4 years $(1994,1996,1998$ and 2001).

Viable recovery path defined using the minimal time of crisis framework developed in this article leads to somewhat softer recovery approaches. The reduction of the fleet size is less stringent and the recovery time is 1 year, which is shorter than in the other scenarii. The recovery strategy associated with the minimum time of crisis requires a shut down of the fishery during one time period (with a negative profit) in order to restore the stock, and then an exploitation pattern making it possible to provide the minimum profit to the whole fleet.

\section{Conclusion}

In this paper, we examine the viability of a fishery with respect to economic, social and biological constraints. The main constraint is a minimum profit per vessel that must be guaranteed at each time period. We show that requiring such a minimum profit induces a minimum threshold for the natural resource, and thus a stronger constraint on the resource stock than the initial biological constraint.

We use the viability approach to determine the set of bioeconomic states and decisions that make it possible to satisfy the constraints dynamically. This set is called the viability kernel of the problem. Any trajectory leaving this set will violate the constraints in a finite time, whatever decisions apply. The system then faces a crisis situation.

We then study transition phases from crisis situations, i.e. states outside the viability kernel, to viable exploitation configurations. These transitions phases are characterized by the time of crisis which is the number of period during which the viability constraints are not met. Of particular interest is the minimal time of crisis with respect to admissible control paths. Such an optimal control problem is original and informative by combining viability and target issues and consequently highlighting the sustainability issues. Nevertheless, this optimal control problem is difficult to address mathematically imposing the use of numerical methods.

To illustrate the approach, we compute recovery paths from the estimated historical crisis of 1994 in the Bay of Biscay Nephrops fishery, based on a simplified model of the fishery. We compare the recovery path given by our approach of minimum time of crisis with the estimated historical path, a sim- 
ulated open access exploitation regime and an economically optimal intertemporal harvesting strategy. Adopting the minimum time of crisis approach, hence the most rapid recovery towards a viable fishery, entails a less stringent reduction in fleet size, the strongest short-term cost to the remaining fishing vessels, but somewhat less variables levels of economic performance during the transition. Final levels of economic performance are however below those observed with the other transition paths, which indicates that adaptation of harvesting strategies according to the status of the fishery may be preferable to single approaches to management in practice. Interestingly, the estimated historical path shows a quite radical reduction in fleet size, with levels of effort per vessel remaining relatively stable in the initial years of the transition phase. This probably illustrates the fact that social constraints as defined in this analysis (in terms of the acceptable speed of fleet reduction) were in fact less stringent than economic viability constraints, with respect to the possible adjustment paths for this modeled fishery.

While these results serve to illustrate the purpose and usefulness of the viability framework for the analysis of fisheries restoration processes, the model does not claim to represent fully the management issues involved in the Bay of Biscay Nephrops fishery. A study of the viability of this fishery through a more realistic and detailed model taking into account the age structure of the resource, environmental uncertainty on recruitment and ecological interactions, is in progress.

\section{A Appendix}

\section{A.1 Parameters of the case study: the Bay of Biscay Nephrops fishery (ICES area VIII)}

The analysis is applied to a case study: the Bay of Biscay Nephrops fishery (ICES area VIII). The numerical model has been calibrated with time-series data available for the fishery.

Biological parameters are estimated using LPUE series (landings per unit of effort) as an index of abundance. We used nonlinear parameter estimation techniques to find the best fit of the predicted LPUE, given the observed LPUE. The fitting criterion is the minimization of the squared deviation between observed and predicted LPUE (Hilborn and Walters, 1992). Figure A.1 represents observed and predicted LPUE. As already stressed, the deterministic surplus production model estimated here does not capture fluctuations in recruitment, and their consequences in terms of the age structure of the stock. In particular, years of high recruitment observed in the late 1980s and early 
2000s have entailed somewhat more variable LPUEs throughout the period, and led to delays in the adjustments observed in the fishery, as compared to those estimated via the model. The estimated dynamics of the bioeconomic system are however qualitatively close to those observed over the period.

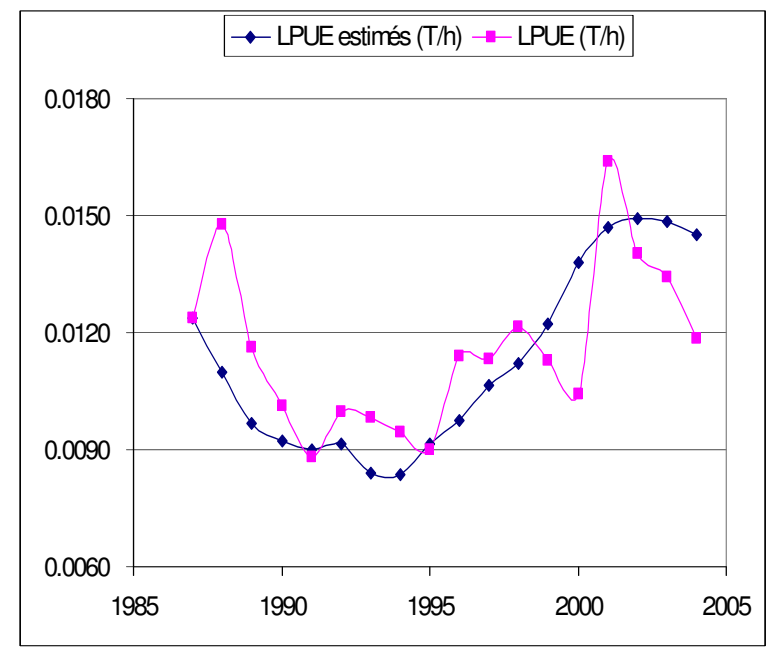

Fig. A.1. Fitting of observed and predicted LPUE in the biological parameters estimation model.

Economic parameters are estimated using costs and earnings data collected by the Fisheries Information System of Ifremer via surveys of individual vessel owners.

Parameters values are as follows.

Parameter value

$$
\begin{aligned}
r & =0.78 \\
B_{\text {sup }} & =30800 \text { tons } \\
q & =72.10^{-7} \mathrm{j}^{-1} \\
p & =8,500 \text { euros per tons } \\
\omega_{f} & =70,000 \text { euros per year } \\
\omega_{v} & =377 \text { euros per day of sea } \\
e_{\text {sup }} & =220 \text { days } \\
\tau_{d} & =33 \% \\
\lambda & =43 \%
\end{aligned}
$$

Constraint level

$$
\begin{aligned}
B_{\min } & =5,000 \text { tons } \\
K_{\text {min }} & =100 \text { vessels } \\
\pi_{\text {min }} & =130,000 \text { euros } \\
\xi_{\text {inf }} & =10 \text { vessels } \\
\xi_{\text {sup }} & =10 \text { vessels }
\end{aligned}
$$


The historical path (with the estimated biomass) is summarized in the following table.

\begin{tabular}{|c|c|c|c|c|c|c|c|c|c|c|}
\hline & 1994 & 1995 & 1996 & 1997 & 1998 & 1999 & 2000 & 2001 & 2002 & 2003 \\
\hline Estimated resource Stock (tons) & 14281 & 15054 & 15482 & 16328 & 16871 & 18082 & 19471 & 20721 & 20728 & 18600 \\
\hline Observed fleet size & 309 & 303 & 291 & 287 & 282 & 270 & 252 & 259 & 245 & 235 \\
\hline $\begin{array}{l}\text { Observed fishing effort } \\
\text { (days at sea per vessel - mean) }\end{array}$ & 164 & 170 & 159 & 161 & 139 & 126 & 123 & 137 & 147 & 163 \\
\hline Profit (keuros per vessel - mean) & 78 & 96 & 91 & 105 & 88 & 87 & 98 & 133 & 148 & 165 \\
\hline
\end{tabular}

\section{A.2 Proofs}

An induced effort constraint: It turns out that a minimum level of effort is required to satisfy the profitability constraint (10). Since catches per unit of effort are proportional to the resource stock under the Schaefer model considered here, this minimum effort level depends on the size of the resource stock.

Lemma 1 The minimum effort $e_{t}$ insuring profit $\pi_{\min }$ at a given level of stock $B_{t}$ is given by

$$
\underline{e}\left(B_{t}\right)=\frac{\pi_{\min }+\omega_{f}}{\frac{p}{\lambda}\left(1-\tau_{d}\right) q B_{t}-\omega_{v}} ;
$$

which leads to the induced constraint

$$
e_{t} \geq \underline{e}\left(B_{t}\right)
$$

Proof of Lemma 1 At a given level of stock biomass $B_{t}$ at time $t$, for constraint (10) to be satisfied, we must have

$$
\left(p\left(1-\tau_{d}\right) q B_{t} e_{t}\right) \frac{1}{\lambda}-\left(\omega_{f}+\omega_{v} e_{t}\right) \geq \pi_{\min }
$$

which leads to

$$
e_{t} \geq \frac{\pi_{\min }+\omega_{f}}{\frac{p}{\lambda}\left(1-\tau_{d}\right) q B_{t}-\omega_{v}}
$$

Hence the minimum effort $\underline{e}\left(B_{t}\right)$.

Note that it is not admissible for this minimum effort to be greater that the maximal effort $e_{\text {sup }}$. As the minimal effort increases as the resource stock decreases, it induces a minimal resource stock.

An induced stock constraint: It turns out that the profit constraint (10) and the maximal effort condition (eq. 8) also generate stronger limitations on stock size than the biological constraint (9). 
Lemma 2 The minimum resource stock for fishing activity to respect the per vessel profit constraint (10) is

$$
\underline{B}=\frac{\pi_{\min }+\left(\omega_{f}+\omega_{v} e_{\mathrm{sup}}\right)}{\frac{p}{\lambda}\left(1-\tau_{d}\right) q e_{\mathrm{sup}}}
$$

which leads to the induced constraint

$$
B_{t} \geq \underline{B}
$$

Proof of Lemma 2 Given the profit equation

$$
\pi_{t}=p q B_{t} e_{t}-\left(\omega_{f}+\omega_{v} e_{t}\right) \geq \pi_{\min }
$$

and combining the minimal effort from Lemma 1 along with the maximum effort bound $e_{\text {sup }}$ (eq. 8), which reads $\underline{e}\left(B_{t}\right) \leq e_{\text {sup }}$, we get

$$
B_{t} \geq \frac{\pi_{\min }+\left(\omega_{f}+\omega_{v} e_{\mathrm{sup}}\right)}{\frac{p}{\lambda}\left(1-\tau_{d}\right) q e_{\mathrm{sup}}}
$$

Hence $\underline{B}$.

Note that at this stock level $\underline{B}$, we have $\underline{e}(\underline{B})=e_{\text {sup }}$, which means that the minimum effort to satisfy the constraint is the maximum effort.

Optimal fishing effort at vessel's level We determine here the effort level that maximizes the profit of vessels.

Lemma 3 If the resource stock is greater than a level $B_{b}=\frac{\omega_{v}}{\frac{p}{\lambda}\left(1-\tau_{d}\right) q}$, the optimal fishing effort of a vessel is its maximum possible effort $e(t)=e_{\text {sup }}$. Else, the optimal effort is 0.

Proof of Lemma 3 The profit, defined by eq. (5) is

$$
\pi_{t}=\left(p\left(1-\tau_{d}\right) q B_{t} e_{t}\right) \frac{1}{\lambda}-\left(\omega_{f}+\omega_{v} e_{t}\right) .
$$

At a given time $t$, and for the resource stock $B_{t}$, taking the profit derivative with respect to the effort level $e_{t}$ leads to

$$
\frac{\partial \pi}{\partial e}=\frac{p}{\lambda}\left(1-\tau_{d}\right) q B_{t}-\omega_{v}
$$

which is positive if the resource stock $B_{t}$ is greater than a threshold $B_{b}$ such that

$$
B_{b}=\frac{\omega_{v}}{\frac{p}{\lambda}\left(1-\tau_{d}\right) q} .
$$


The maximization of the individual instantaneous profit thus would lead to an effort following a "bang-bang" strategy: no fishing if $B_{t}<B_{\mathrm{b}}$ and a maximum activity $e_{\text {sup }}$ if $B_{t}>B_{b}$. In our illustrative case, this value is $B_{\mathrm{b}}=4,075$ tons, which is lower than the resource constraint $B_{\min }$. We will thus consider that it would always be optimal to fish as much as possible if there is no regulation of the fishery.

\section{A.3 Stationary states}

Stationary states: Among all viable states, particular states allow the dynamics to follow stationary trajectories for associated ad hoc exploitation decisions. These stationary states are characterized by $B_{t+1}=B_{t}$ and $K_{t+1}=K_{t}$ which leads to $\xi_{t}=0$ and $R_{t}=C_{t}$. This last statement is equivalent to $e_{t} K_{t}=\frac{r}{q}\left(1-\frac{B_{t}}{B_{\text {sup }}}\right)$. Such a relation induces admissible stationary states whenever all the constraints hold true. Extreme cases correspond to maximum effort $e_{\text {sup }}$ on the one hand (which leads to a linear relationship between the fleet size and the resource stock), and minimum effort $\underline{e}\left(B_{t}\right)$ on the other hand. Hence we obtain the conditions on fleet size and resource stock

$$
\frac{r}{q \underline{e}(B)}\left(1-\frac{B}{B_{\text {sup }}}\right) \leq K \leq \frac{r}{q e_{\text {sup }}}\left(1-\frac{B}{B_{\text {sup }}}\right)
$$

which can occur if stock $B$ is larger than $\underline{B}$. These two frontiers are represented on Fig. 1. The inner area corresponds to possible stationary states that satisfy all the constraints, including the profitability constraint.

Among the set of stationary states, some are of particular interest as usual reference points in the literature:

- The Open Access Equilibrium (OAE) corresponds to the equilibrium stationary state reached by a fishery under open access, with vessels able to freely enter or leave the fishery, and choose their effort level, in response to changes in profit levels in comparison to constraint (10).

- The Maximum Economic Yield (MEY) corresponds to the stationary state in which the total profit of the fleet is maximum. This implies a resource stock for which the marginal productivity in value of the resource stock equals the marginal costs of catch, and to a production structure that minimizes costs, i.e. a minimum fleet size given that there are fixed costs and a positive productivity of effort.

- The Maximum Sustainable Yield (MSY) corresponds to the resource stock associated with the largest stock regeneration $R\left(B_{M S Y}\right)$. Various stationary states are possible here, depending on fleet size and effort, and the associated per-vessel catches (total catches being constant). 
Note that there is a maximum sustainable size for the fleet. This corresponds to the same stock size as in the MEY state, but with a maximum number of vessels sharing the global effort, the minimum profit constraint (10) being respected.

Acknowledgments This paper was prepared as part of the CHALOUPE research project, funded by the French National Research Agency under its Biodiversity program. The authors would like to thank Olivier Guyader, Claire Macher, Michel Bertignac and Fabienne Daurès for their assistance in the development of the simplified bioeconomic model of the bay of Biscay nephrops fishery used for the analysis, and for the fruitful discussions regarding the application of viability analysis to the problem of fisheries restoration.

\section{References}

Aubin, J.-P., 1991. Viability theory. Birkhauser, Springer Verlag.

Beddington, J.R., and May, R.M., 1977. Harvesting natural populations in a randomly fluctuating environment. Science, 197:463-465.

Béné, C., and Doyen, L., 2000. Storage and Viability of a Fishery with Resource and Market Diphased Seasonalities. Journal of Environmental Resource Economics, 15:1-26.

Béné, C., Doyen, L. and Gabay, D., 2001. A viability analysis for a bioeconomic model. Ecological Economics, 36:385-396.

Bjorndal, T., and Conrad, J.M., 1987. The Dynamics of an Open Access Fishery. The Canadian Journal of Economics / Revue canadienne d'Economique, 20:74-85.

Clark, C.W., 1976. Mathematical bioeconomics: the optimal management of renewable resources. Wiley-Interscience publication.

Clark, C.W., 1985. Bioeconomic Modelling and Fisheries Management. John Wiley and Sons: New York.

Clark, C.W., 1990. Mathematical Bio-economics: the optimal management of renewable resources. Second edition, John Wiley and Sons: New York.

Clark, C. and Munro, G., 1975. The Economics of Fishing and Modern Capital Theory: A Simplified Approach. Journal of Environmental Economics and Management, 2:92-106.

Clark, C.W., Clarke, F.H., and Munro,G., 1979. The optimal management of renewable resource stocks: problems of irreversible investment. Econometrica, 47:25-47.

Cury, P., Mullon, C., Garcia, S. and Shannon, L.J., 2005. Viability theory for an ecosystem approach to fisheries. ICES Journal of Marine Science, 62:577-584.

De Lara, M., Doyen, L., Guilbaud, T., and Rochet, M.J., in press. Is a man- 
agement framework based on spawning stock biomass indicator sustainable? A viability approach. Marine science.

Doyen, L. and Béné, C., 2003. Sustainability of fisheries through marine reserves: a robust modeling analysis. Journal of Environmental Management, 69:1-13.

Doyen, L. and Saint-Pierre, P., 1997. Scale of viability and minimum time of crisis. Set-valued Analysis, 5:227-246.

Eisenack, K., Sheffran, J. and Kropp, J., 2006. The Viability Analysis of Management Frameworks for fisheries. Environmental modelling and assessment, 11:69-79.

Food and Agriculture Organization, 2004. The state of World Fisheries and Aquaculture. FAO, Sofia.

Garcia, S. and Grainger, J.R., 2005. Gloom and doom? The future of marine capture fisheries. Phil. Trans. R. Soc. B., 360:21-46.

Gordon, H.S., 1954. The economic theory of a common property resource: the fishery. Journal of Political Economy, 82:124-142.

Hilborn, R. and Walters, C.J., 1992. Quantitative Fisheries Stock Assessment: Choice, Dynamics and Uncertainty. Chapman and Hall, New York.

Holland, D. S., 2000. A bioeconomic model for marine sanctuaries on Georges Bank. Canadian Journal of Fisheries and Aquatic Sciences, 57:13071319.

Homans, F., and Wilen, J.E., 1997. A Model of Regulated Open Access Resource Use. Journal of Environmental Economics and Management, 32:1-21.

Mardle, S. and Pascoe, S., 2002. Modelling the effects of trade-offs between long run and short run objectives in the North Sea. Journal of Environmental Management, 65:49-62.

Martinet, V. and Doyen, L., 2007. Sustainability of an economy with an exhaustible resource: a viable control approach. Resource and Energy Economics, 29:17-39.

McKelvey, R., 1985. Decentralized regulation of a common-property resource industry with irreversible investment. Journal of Environmental Economics and Management, 12:298-307.

Morris, W.F. and Doak, D.F., 2003. Quantitative Conservation Biology: Theory and Practice of Population Viability Analysis. Sinauer Associates.

Schaefer, M.B., 1954. Some aspects of the dynamics of populations. Bull. Int. Am. Trop. Tuna Comm., 1:26-56.

Smith, V.L., 1968. Economics of Production from Natural Resources. American Economic Review, 58:409-431.

Smith, V.L., 1969. On Models of Commercial Fishing. Journal of Political Economy, 77:181-196.

Wilen, J., 1976. Common Property Resources and the Dynamics of Overexploitation. UBC Working Paper n3.

Ward, J.M., and Sutinen, J.G., 1994. Vessel Entry-Exit Behavior in the Gulf of Mexico Shrimp Fishery. American Journal of Agricultural Economics, 76:916-923. 